\title{
Multi-Instantons in Higher Dimensions and Superstring Solitons
}

\author{
Eugene K. LOGINOV
}

Department of Physics, Ivanovo State University, 39 Ermaka Str., Ivanovo, 153025 Russia

E-mail: loginov@ivanovo.ac.ru

Received June 30, 2005; Published online August 16, 2005

Original article is available at http://www.emis.de/journals/SIGMA/2005/Paper002/

\begin{abstract}
We construct octonionic multi-instantons for the eight and seven dimensional Yang-Mills theory. Extended soliton solutions to the low-energy heterotic field theory equations of motion are constructed from these octonionic multi-instantons. The solitons describe a string in ten-dimensional Minkowski space, and preserve one and two of the sixteen space-time supersymmetries correspondingly.
\end{abstract}

Key words: multi-instantons; supersymmetric solitons

2000 Mathematics Subject Classification: 83E30

\section{Introduction}

We search solutions for lowest nontrivial order in $\alpha^{\prime}$ of the equations of motion that follow from the bosonic action

$$
S=\frac{1}{2 k^{2}} \int d^{10} x \sqrt{-g} e^{-2 \phi}\left(R+4(\nabla \phi)^{2}-\frac{1}{3} H^{2}-\frac{\alpha^{\prime}}{30} \operatorname{Tr} F^{2}\right) .
$$

We are interested in solutions that preserve at least one supersymmetry. This requires existence in ten dimensions of at least one Majorana-Weyl spinor $\epsilon$ such that the supersymmetry variations of the fermionic fields vanish for such solutions

$$
\begin{aligned}
& \delta \chi=F_{M N} \Gamma^{M N} \epsilon, \\
& \delta \lambda=\left(\Gamma^{M} \partial_{M} \phi-\frac{1}{6} H_{M N P} \Gamma^{M N P}\right) \epsilon, \\
& \delta \psi_{M}=\left(\partial_{M}+\frac{1}{4} \Omega_{M}^{A B} \Gamma_{A B}\right) \epsilon,
\end{aligned}
$$

where $\chi, \lambda$ and $\psi_{M}$ are the gaugino, dilatino and the gravitino fields, respectively. The generalized spin connection $\Omega$ is a non-Riemannian connection related to the spin connection $\omega$ and the anti-symmetric tensor field strength $H$ by

$$
\Omega_{M}^{A B}=\omega_{M}^{A B}-H_{M}^{A B} .
$$

In [1] a one-brane solution of heterotic theory which is an everywhere smooth solution of the equations of motion was found. Construction of this solution involves essentially properties of octonions. One of the many bizarre features of this soliton is that it preserves only one of the sixteen space-time supersymmetries, in contrast to previously known examples of supersymmetric solitons which all preserve half of the supersymmetries. In [2] a two-brane solution of heterotic theory was found. This soliton preserves two of the sixteen supersymmetries and hence corresponds to $N=1$ space-time supersymmetry in $(2+1)$ dimensions transverse to the seven dimensions where the Yang-Mills instanton is defined. 


\section{Multi-instantons in eight dimensions}

We start by picking a particular commuting $S O(8)$ spinor $\eta$ with $\Gamma_{9} \eta=\eta$ normalized to $\eta^{T} \eta=1$. Then we can introduce a fourth-rank antisymmetric Spin(7)-invariant tensor

$$
f_{m n p s}=-\eta^{T} \Gamma_{m n p s} \eta .
$$

There exists an explicit construction of the $S O(8)$ gamma matrices in terms of the octonion structure constants $c_{i j k}$ defined by

$$
e_{i} e_{j}=-\delta_{i j}+c_{i j k} e_{k}
$$

where $c_{i j k}$ are antisymmetric in $(i, j, k)$, nonzero and equal to unity for the seven combinations (123), (145), (167), (246), (275), (374), (365). Using this construction and an explicit choice for $\eta$ can find

$$
f_{i j k 8}=c_{i j k}, \quad f_{i j k l}=\delta_{i l} \delta_{j k}-\delta_{i k} \delta_{j l}+c_{i j r} c_{k l r} .
$$

Suppose $e_{p s}$ are the standard generators of the Lie algebra so(8). Define elements of the subalgebra $s o(7)$ of $s o(8)$ by

$$
E_{m n}=\frac{1}{8}\left(3 \delta_{m p} \delta_{n s}-3 \delta_{m s} \delta_{n p}-f_{m n p s}\right) e_{p s} .
$$

Consider now the Yang-Mills gauge theory in eight dimensions with the gauge group Spin(7). We proceed from the following ansatz

$$
A_{m}=\frac{4}{3} \frac{\lambda^{\dagger} y^{n}}{\left(1+y^{\dagger} y\right)} E_{m n}
$$

where $y$ is a column vector with the octonions $y_{1}, \ldots, y_{N}$ such that

$$
\begin{aligned}
y^{\dagger} & =\left(y_{1}^{k}, \ldots, y_{N}^{k}\right) \bar{e}_{k}, \quad y_{I}^{k} \in \mathbb{R}, \\
\lambda^{\dagger} & =\left(\lambda_{1}, \ldots, \lambda_{N}\right), \quad \lambda_{I} \in \mathbb{R}^{+}, \\
y_{I}^{k} & =\left(b_{I}^{k}+x^{k}\right) \lambda_{I} .
\end{aligned}
$$

Using the switching relations for the generators $E_{m n}$, we get the self-dual field strength

$$
F_{m n}=-\frac{4}{9} \lambda^{\dagger}\left[\frac{3\left(2+2 y^{\dagger} y-y^{i} y_{i}^{\dagger}\right) E_{m n}+\left(3 \delta_{m i} \delta_{n s}-3 \delta_{m s} \delta_{n i}-f_{m n i s}\right) E_{s j} y^{j} y_{i}^{\dagger}}{\left(1+y^{\dagger} y\right)^{2}}\right] \lambda .
$$

Thus we have a Spin(7)-invariant solution of the Yang-Mills field equations which depends on at most $9 N$ effective parameters (cf. [1]).

\section{Superstring solitons}

Now we denote world indices of the eight-dimensional space transverse to the 1-brane by $\mu, \nu=$ $1, \ldots, 8$ and the corresponding tangent space indices by $m, n=1, \ldots, 8$. We assume that no fields depend on the longitudinal coordinates and that the nontrivial tensor fields in the solution have only transverse indices. Then the gamma matrix terms in (1) are sensitive only to the $\operatorname{Spin}(7)$ part of $\epsilon$. Thus taking $\epsilon$ to be a $\operatorname{Spin}(7)$ singlet $\eta$ and the non-vanishing components of $F_{M N}$ to be those given by the eight dimensional octonionic multi-instanton the supersymmetry variation $\delta \chi$ of the gaugino vanishes. This follows from the fact that

$$
\left(3 \delta_{m p} \delta_{n s}-3 \delta_{m s} \delta_{n p}-f_{m n p s}\right) \Gamma^{p s} \eta=0
$$


and self-duality of $F_{\mu \nu}$. To deal with the other supersymmetry variations, we must adopt an ansatz for the non-trivial behavior of the metric and antisymmetric tensor fields in the eight dimensions transverse to the 1-brane. Let

$$
g_{\mu \nu}=e^{(6 / 7) \phi} \delta_{\mu \nu}, \quad H_{\mu \nu \lambda}=\frac{1}{7} f_{\mu \nu \lambda \sigma} \partial^{\sigma} \phi
$$

where $\phi$ is to be identified with the dilaton field. With this ansatz, we can prove that it suffices to take $\epsilon$ to be a constant $\operatorname{Spin}(7)$ invariant spinor to make the dilatino variation $\delta \lambda$ and the gravitino variation $\delta \psi_{M}$ vanish.

In order to determine the solution completely, we need only to solve the Bianchi identity

$$
d H=\alpha^{\prime}\left(\operatorname{tr} R \wedge R-\frac{1}{30} \operatorname{Tr} F \wedge F\right),
$$

where Tr refers to the trace in the adjoint representation of $E_{8}$ or $S O(32)$ in the corresponding heterotic string theory. For order $\alpha^{\prime}$ we can neglect the first term and obtain the following dilaton solution:

$$
e^{-(6 / 7) \phi}=e^{-(6 / 7) \phi_{0}}+\frac{32}{9} \frac{\lambda^{\dagger}\left(2+2 y^{\dagger} y-y^{i} y_{i}^{\dagger}\right) \lambda}{\left(1+y^{\dagger} y\right)^{2}} .
$$

The metric and antisymmetric fields are constructed of this dilaton field according to the spacetime-supersymmetric ansatz. However, the metric and the field strength fall of only as $1 / r^{2}$ and this implies that the ADM mass per unit length of this string diverges to be just like the mono-instanton solution (see [1]).

\section{Multi-instantons in seven dimensions}

Consider now the Yang-Mills gauge theory in seven dimensions with the gauge group $G_{2}$. This group may be defined as the group of all automorphisms of the algebra of octonions $\mathbb{O}$. The corresponding Lie algebra $g_{2}$ is generated by all derivations of the form

$$
D_{i j}: z \rightarrow \frac{1}{6}\left[\left[e_{i}, e_{j}\right], z\right]+\frac{1}{2}\left(e_{i}, e_{j}, z\right)
$$

where the associator $(x, y, z)=(x y) z-x(y z)$ is skew-symmetric for $x, y, z \in \mathbb{O}$. We can then introduce a completely antisymmetric tensor $c_{i j k l}$ by

$$
\left(e_{i}, e_{j}, e_{k}\right)=2 c_{i j k l} e_{l} .
$$

It is easy to prove that this tensor is $G_{2}$-invariant. We choose the ansatz

$$
A_{m}=\frac{3}{2} \frac{\lambda^{\dagger} y^{n}}{\left(1+y^{\dagger} y\right)} D_{m n}
$$

where $y$ is the above-mentioned column vector of octonions.

Using the switching relations for the derivations $D_{m n}$, we get the self-dual field strength

$$
F_{m n}=-\frac{3}{4} \lambda^{\dagger}\left[\frac{2\left(2+2 y^{\dagger} y-y^{i} y_{i}^{\dagger}\right) D_{m n}+\left(2 \delta_{m i} \delta_{n s}-2 \delta_{m s} \delta_{n i}-c_{m n i s}\right) D_{s j} y^{j} y_{i}^{\dagger}}{\left(1+y^{\dagger} y\right)^{2}}\right] \lambda .
$$

Thus we have a $G_{2}$-invariant solution of the Yang-Mills field equations which depends on at most $8 N$ effective parameters. In fact we obtain $E_{6}$-invariant solution of the equations. Indeed, $E_{6}$ is a group of linear transformations of the exceptional Jordan algebra $J(\mathbb{O})$ which preserve 
a cubic form (norm) $n(X)$ of any element $X \in J(\mathbb{O})$. It can be shown that there exists a set of elements $X_{i j k l}=X\left(e_{i}, e_{j}, e_{k}, e_{l}\right)$ of $J(\mathbb{O})$ such that the norm

$$
n\left(X_{i j k l}\right)=c_{i j k l} \text {. }
$$

Since the group $G_{2}$ can be isomorphically enclosed into the group $E_{6}$, we prove the $E_{6}$-invariance of the found solution.

\section{Superstring solitons}

Let us now show that this solution can be extended to a soliton solution of the heterotic string. To obtain this we choose $\epsilon$ to be a constant $G_{2}$ invariant spinor, and the metric and antisymmetric tensor fields to be of the form

$$
g_{\mu \nu}=e^{\phi} \delta_{\mu \nu}, \quad H_{\mu \nu \lambda}=\frac{1}{4} f_{\mu \nu \lambda \sigma} \partial^{\sigma} \phi .
$$

To order $\alpha^{\prime}$ we obtain the following dilaton solution:

$$
e^{-\phi}=e^{-\phi_{0}}+\frac{4 \lambda^{\dagger}\left(2+2 y^{\dagger} y-y^{i} y_{i}^{\dagger}\right) \lambda}{\left(1+y^{\dagger} y\right)^{2}} .
$$

Obviously, this solution also does not have finite energy per unit length, and therefore it complicates physical implications. However, as in the mono-instanton case (see [2]), we can suppose that this divergent energy is an infrared phenomenon and does not preclude the existence of a well-behaved low-energy effective action governing the string dynamics on scales large relative to its core size.

Remark 1. In eight and seven dimensions, in contrast to four dimensions, the Yang-Mills action is infinite and the topological meaning of the solutions is unknown. In the case we cannot assert that these solutions are gauge equivalent to the mono-instanton ones that were found in previous papers. They are new solutions which depend on at most $9 N$ and $8 N$ effective parameters correspondingly.

Remark 2. The fact that these solutions do not have finite action involves that the corresponding supersymmetric solutions do not have finite energy per unit length. This is a characteristic feature of such solutions. There are no finite-action solutions of the Yang-Mills equations in eight and seven dimensions. In order to get finite-action Yang-Mills equations one would "compactify" some of the dimensions.

Remark 3. These notes are based on my lecture at the Sixth International Conference "Symmetry in Nonlinear Mathematical Physics" (June 20-26, 2005, Kyiv, Ukraine). More detailed version of these notes can be found in $[3,4]$.

\section{Acknowledgements}

Research supported by RFBR Grant 04-02-16324.

[1] Harvey J.A., Strominger A., Octonionic superstring solitons, Phys. Rev. Lett., 1991, V.66, 549-552.

[2] Gunaydin M., Nicolai H., Seven dimensional octonionic Yang-Mills instantons and its extension to an heterotic string soliton, Phys. Lett. B, 1995, V.351, 169-172.

[3] Loginov E.K., Multi-instantons in seven dimensions, J. Math. Phys., 2005, V.46, 063506, 6 pages.

[4] Loginov E.K., Multi-instantons and superstring solitons, Phys. Lett. B, 2005, V.618, 265-268. 